\title{
Recent results on the algebraic approach to the CSP
}

\author{
Andrei A. Bulatov ${ }^{1}$ and Matthew A. Valeriote ${ }^{2}$ \\ 1 School of Computing Science \\ Simon Fraser University \\ Burnaby, BC, Canada, V5A 1S6 \\ abulatov@cs.sfu.ca \\ 2 Department of Mathematics \& Statistics \\ McMaster University \\ Hamilton, Ontario, Canada L8S 4K1 \\ matt@math.mcmaster.ca
}

\begin{abstract}
We describe an algebraic approach to the constraint satisfaction problem (CSP) and present recent results on the CSP that make use of, in an essential way, this algebraic framework.
\end{abstract}

\section{Introduction}

This paper presents material from the talks that the authors gave at the Dagstuhl seminar on the Complexity of Constraints held in 2006. The primary goals of the talks were to describe an algebraic approach to the constraint satisfaction problem and to present, within the algebraic context, recent results relating to two of the main motivating conjectures in the field.

During our talks, by necessity, a fair amount of time was occupied in describing basic and advanced universal algebra. In particular, overviews of two approaches to analyzing the local structure of finite algebras were given. The first, known as tame congruence theory, was developed in the 1980s by David Hobby and Ralph McKenzie and has played an important role in the development of universal algebra ever since. The second is a much more recent approach developed by Bulatov specifically to address questions relating to the CSP. For readers who wish to learn more about basic universal algebra we recommend [17] and [37]. For more information on tame congruence, the works [27] or [19] can be consulted. The paper [10] contains details of the theory developed by Bulatov.

\section{Constraint Satisfaction and Algebra}

\subsection{Constraint Satisfaction}

We use the homomorphism definition of the CSP. A vocabulary $\tau$ is a finite set of relational symbols; each symbol has an associated arity. A (finite) relational structure $\mathcal{H}$ with vocabulary $\tau$ consists of a finite set $H$, its universe, and, for 
every relational symbol $R \in \tau$ of arity $n$, an $n$-ary relation $R^{\mathcal{H}}$ on $H$, the interpretation of $R$ by $\mathcal{H}$. A homomorphism of a structure $\mathcal{G}$ to a structure $\mathcal{H}$ with the same vocabulary $\tau$ is a mapping $\varphi: G \rightarrow H$ from the universe of $\mathcal{G}$ to the universe of $\mathcal{H}$ such that for each ( $n$-ary) relational symbol $R \in \tau$ and any tuple $\left(a_{1}, \ldots, a_{n}\right) \in R^{\mathcal{G}}$ the tuple $\left(\varphi\left(a_{1}\right), \ldots, \varphi\left(a_{n}\right)\right)$ belongs to $R^{\mathcal{H}}$.

For a finite structure $\mathcal{H}$ the non-uniform constraint satisfaction problem, denoted $\operatorname{CSP}(\mathcal{H})$, is the following combinatorial problem: Given a structure $\mathcal{G}$ of the same vocabulary as $\mathcal{H}$, decide whether or not there is a homomorphism from $\mathcal{G}$ to $\mathcal{H}$. The structure $\mathcal{H}$ is called the template, and $\mathcal{G}$ is called the instance. For a class $\mathfrak{H}$ of relational structures, in the uniform constraint satisfaction problem, denoted $\operatorname{CSP}(\mathfrak{H})$, the question is: given a structure $\mathcal{H} \in \mathfrak{H}$ and a structure $\mathcal{G}$ over the same vocabulary as $\mathcal{H}$, decide whether there exists a homomorphism from $\mathcal{G}$ to $\mathcal{H}$. Sometimes it is convenient to think of a uniform problem as of the union or collection of non-uniform problems $\operatorname{CSP}(\mathcal{H})$ for $\mathcal{H} \in \mathfrak{H}$.

\section{Example 1 (NAE, Linear Equations, and H-Colouring).}

1. Let $\mathcal{H}_{N A E}$ be a relational structure with universe $\{0,1\}$ and one ternary relation $R^{\mathcal{H}_{N A E}}=\{0,1\}^{3} \backslash\{(0,0,0),(1,1,1)\}$. It is easy to see that the problem $\operatorname{CSP}\left(\mathcal{H}_{N A E}\right)$ is the same as the Not-ALL-EQUal SATISFIABILITY problem, in which, given a set of propositional variables and a set of triples of these variables, the question is whether or not it is possible to assign values to the variables such that the variables from each of the specified triples take both possible values, 0 and 1 .

2. Let $F$ be a finite field and $\Gamma$ the set of all relations over $F$ that can be represented as the set of solutions of a linear equation over $F$. Let $\mathfrak{H}_{L Q}(F)$ denote the set of all structures with universe $F$, whose relations are from $\Gamma$. Then the uniform problem $\operatorname{CSP}\left(\mathfrak{H}_{L Q}(F)\right)$ is equivalent in a certain sense to the problem of solving systems of linear equations over $F .^{3}$

3 . Let $H$ be a (directed) graph. In the $H$-ColOURING problem we are asked whether there is a homomorphism from a given graph $G$ to $H$. So, the $H$ Colouring problem is just the problem $\operatorname{CSP}(H)$.

Two major issues have arisen in the study of the study of the constraint satisfaction problem. The first one is the computational complexity of solving such problems. Although constraint satisfaction problems may belong to and be

\footnotetext{
${ }^{3}$ The size of a CSP instance is defined to be the length of a reasonable encoding of the structures involved, that is the instance in the case of a non-uniform problem, and the source structure and the template in the case of a uniform problem. Usually such an encoding includes a list of elements of the structures and a list of tuples in all relations. In some cases such a general representation is not the most natural. For example, the natural representation of a $\operatorname{CSP}\left(\mathfrak{H}_{L Q}(F)\right)$ instance is a list of equations defining relations of the template. Although no example is known, different representation may affect the complexity of uniform problems. However, for the sake of generality throughout the paper we use the explicit representation of relational structures. The choice of representation does not affect the complexity of non-uniform problems.
} 
complete in many complexity classes, see, e.g. [1,33,34], in this paper we concentrate on problems solvable in polynomial time (such problems are often said to be tractable). The remaining problems are called intractable. (Throughout the paper we assume $\mathrm{P} \neq \mathrm{NP}$.) All the intractable problems known so far turn out to be NP-complete. This prompted Feder and Vardi [24] to suggest the Dichotomy Conjecture: Every non-uniform CSP is either tractable or NP-complete.

The second issue is the descriptive complexity of non-uniform problems. Let $\mathcal{H}$ be a relational structure. The class of structures homomorphic to $\mathcal{H}$ is often denoted by $\operatorname{CSP}(\mathcal{H})$ (this does not cause any confusion, because $\operatorname{CSP}(\mathcal{H})$ is the class of yes-instances of the corresponding constraint satisfaction problem, and therefore the language associated to this problem). In many cases the class $\operatorname{CSP}(\mathcal{H})$ can be characterized as the class of all structures satisfying some formula in a certain logic. The goal is to describe structures $\mathcal{H}$ such that $\operatorname{CSP}(\mathcal{H})$ is expressible in this logic. We concentrate on the logic corresponding to Datalog. For definitions of Datalog, Datalog expressibility, related properties of structures and problems, as well as results on other important logical languages the reader is referred to [16] from the same volume.

Example 2 (continued).

1. NAE is NP-complete, [39].

2. Linear Equations is not expressible in Datalog, [24].

3. $H$-COLORING is tractable if and only if $H$ is a bipartite graph. In this case it is expressible in Datalog. Otherwise it is NP-complete, [26].

\subsection{Polymorphisms and Algebras}

In this section we provide a brief overview of the algebraic approach to the constraint satisfaction problem.

At the core of this approach is the concept of a polymorphism. Let $R$ be a relation on a set $A$. An ( $n$-ary) operation $f$ on the same set is said to be a polymorphism of $R$ if for any tuples $\mathbf{a}_{1}, \ldots, \mathbf{a}_{n} \in R$ the tuple $f\left(\mathbf{a}_{1}, \ldots, \mathbf{a}_{n}\right)$ obtained by applying $f$ component-wise also belongs to $R$. The relation $R$ is called an invariant of $f$. An operation $f$ is a polymorphism of a relational structure $\mathcal{H}$ if it is a polymorphism of each relation of the structure. The set of all polymorphisms of $\mathcal{H}$ is denoted by $\operatorname{Pol}(\mathcal{H})$. For a collection $C$ of operations $\operatorname{Inv}(C)$ denotes the set of invariants of all operations from $C$.

Example 3 ([40]). Let $R$ be the solution space of a system of linear equations over a finite field $F$. Then the operation $m(x, y, z)=x-y+z$ is a polymorphism of $R$. Indeed, let $A \cdot \mathbf{x}=\mathbf{b}$ be the system defining $R$, and $\mathbf{x}, \mathbf{z}, \mathbf{y} \in R$. Then

$$
A \cdot m(\mathbf{x}, \mathbf{z}, \mathbf{y})=A \cdot(\mathbf{x}-\mathbf{z}+\mathbf{y})=A \cdot \mathbf{x}-A \cdot \mathbf{z}+A \cdot \mathbf{y}=\mathbf{b}-\mathbf{b}+\mathbf{b}=\mathbf{b} .
$$

In fact, the converse can also be shown: if $R$ is invariant under $m$ then it is the solution space of a certain system of linear equations. 
The following theorem relates polymorphisms, complexity, and expressibility in Datalog

Theorem $1([\mathbf{2 9}, \mathbf{3 1}, \mathbf{3 5}])$. Let $\mathcal{H}_{1}$ and $\mathcal{H}_{2}$ be two structures with a common universe.

1. If $\operatorname{Pol}\left(\mathcal{H}_{1}\right) \subseteq \operatorname{Pol}\left(\mathcal{H}_{2}\right)$ then $\operatorname{CSP}\left(\mathcal{H}_{2}\right)$ is log-space reducible to $\operatorname{CSP}\left(\mathcal{H}_{1}\right)$.

2. If $\operatorname{Pol}\left(\mathcal{H}_{1}\right) \subseteq \operatorname{Pol}\left(\mathcal{H}_{2}\right)$ and $\operatorname{CSP}\left(\mathcal{H}_{1}\right)$ is expressible in Datalog, then $\operatorname{CSP}\left(\mathcal{H}_{2}\right)$ is expressible in Datalog.

An algebra is a pair $\mathbb{A}=(A ; F)$ consisting of a set $A$, the universe of $\mathbb{A}$, and a set $F$ of operations on $A$, the basic operations of $\mathbb{A}$. Operations that can be obtained from the basic operations of $\mathbb{A}$ and the projection operations on $A$, that is operations of the form $f\left(x_{1}, \ldots, x_{n}\right)=x_{i}$, by means of compositions are called term operations of $\mathbb{A}$. Term $(\mathbb{A})$ denotes the set of all term operations of $\mathbb{A}$. Operations that can be obtained from term operations by substituting constants are called polynomial operations (or just polynomials) of $\mathbb{A}$.

Any relational structure $\mathcal{H}$ and therefore any non-uniform constraint satisfaction problem can be associated with an algebra $\operatorname{Alg}(\mathcal{H})=(H ; \operatorname{Pol}(\mathcal{H}))$ where $H$ is the universe of $\mathcal{H}$. Conversely, any algebra $\mathbb{A}=(A ; F)$ corresponds to a class of structures $\operatorname{Str}(\mathbb{A})$ that includes all the structures $\mathcal{H}$ with universe $A$, having a finite vocabulary, and such that $\operatorname{Term}(\mathbb{A}) \subseteq \operatorname{Pol}(\mathcal{H})$. Therefore every algebra gives rise to a uniform constraint satisfaction problem $\operatorname{CSP}(\operatorname{Str}(\mathbb{A}))$, which we will denote by $\operatorname{CSP}(\mathbb{A})$.

An algebra $\mathbb{A}$ is called tractable if $\operatorname{CSP}(\mathcal{H})$ is tractable for each $\mathcal{H} \in \operatorname{Str}(\mathbb{A})$ and is called $N P$-complete if $\operatorname{CSP}(\mathcal{H})$ for some $\mathcal{H} \in \operatorname{Str}(\mathbb{A})$ is. Theorem 1 implies that if $\operatorname{CSP}(\mathcal{H})$ is tractable then the algebra $\operatorname{Alg}(\mathcal{H})$ is tractable. We make two observations. First, if an algebra $\mathbb{A}$ is not tractable, it does not mean that $\operatorname{CSP}(\mathcal{H})$ is intractable for all $\mathcal{H} \in \operatorname{Str}(\mathbb{A})$; this class always contains poor structures whose associated class of constraint satisfaction problems is very easy. Second, if $\mathbb{A}$ is tractable it does not necessarily mean that the uniform problem $\operatorname{CSP}(\mathbb{A})$ is tractable. Although no example is known, it may be the case that the time complexity of problems $\operatorname{CSP}(\mathcal{H}), \mathcal{H} \in \operatorname{Str}(\mathbb{A})$, does not have a uniform polynomial bound, even though the complexity of each problem is polynomially bounded. To distinguish these two potential situations we sometimes call tractable algebras locally tractable and algebras for which $\operatorname{CSP}(\mathbb{A})$ is tractable, globally tractable. In other words, $\mathbb{A}$ is locally tractable if every non-uniform problem from $\operatorname{CSP}(\mathbb{A})$ is solvable in polynomial time.

The relational width of an algebra $\mathbb{A}$ is a parameter related to certain properties of Datalog programs or propagation algorithms that solve the problems $\operatorname{CSP}(\mathcal{H})$ for $\mathcal{H} \in \operatorname{Str}(\mathbb{A})$. The algebra $\mathbb{A}$ is said to be of bounded width if $\operatorname{CSP}(\mathcal{H})$ is expressible in Datalog for any structure $\mathcal{H} \in \operatorname{Str}(\mathbb{A})$. For complete definitions and discussion of this concept see [16] in the same volume.

The tractability and relational width of an algebra usually follows from the presence of a certain polymorphism of a structure (or a term operation of an algebra). 
Example 4 ([5, 13, 20, 22, 29, 31]). If one of the following operations is a term operation of an algebra $\mathbb{A}$ [a polymorphism of a relational structure $\mathcal{H}$ ] then $\operatorname{CSP}(\mathbb{A})[\operatorname{CSP}(\mathcal{H})]$ is tractable:

- a semilattice operation, that is a binary operation $f$ satisfying the equations: (a) $f(x, x) \approx x$ (idempotency); (b) $f(x, y) \approx f(y, x)$ (commutativity); (c) $f(f(x, y), z) \approx f(x, f(y, z))$ (associativity);

- a 2-semilattice operation, that is a binary operation $f$ satisfying the equations $f(x, x) \approx x$, $f(x, y) \approx f(y, x)$, and $f(x, f(x, y)) \approx f(x, y)$

- a near-unanimity $(N U)$ operation, that is an operation $f$ satisfying the equations $f(x, \ldots, x, y) \approx f(x, \ldots, x, y, x) \approx \ldots \approx f(y, x, \ldots, x) \approx x$.

- a majority operation, that is a ternary operation $g$ satisfying the equations $g(x, x, y) \approx g(x, y, x) \approx g(y, x, x) \approx x$ (thus a majority operation is a ternary near-unanimity operation).

- a Mal'tsev operation, that is a ternary operation $h$ satisfying the equations $h(x, x, y) \approx h(y, x, x) \approx y$.

- a generalized majority-minority (GMM) operation, that is an operation $f$ such that for any $a, b \in A$ one of the following 2 conditions holds:

$f(x, \ldots, x, y)=f(x, \ldots, x, y, x)=\ldots=f(y, x, \ldots, x)=x$, for $x, y \in\{a, b\}$; or

$f(x, \ldots, x, y)=f(y, x, \ldots, x)=x$, for $x, y \in\{a, b\}$.

Example 5. If one of the following operations is a polymorphism of a relational structure $\mathcal{H}$, then $\operatorname{CSP}(\mathcal{H})$ is expressible in Datalog:

- a semilattice operation;

- a 2-semilattice operation;

- a near-unanimity operation;

- a majority operation.

On the other hand, the intractability of a relational structure (or an algebra) seems to imply that it has rather uninteresting polymorphisms (term operations, respectively). An operation $f$ on a set $A$ is said to be an essentially unary surjective operation if $f\left(x_{1}, \ldots, x_{n}\right)=g\left(x_{i}\right)$ for some $i$ and some surjective map $g(x)$ of $A$.

Example 6 (continued).

1. An operation $f$ is a polymorphism of $\mathcal{H}_{N A E}$ if and only if $f$ is an essentially unary surjective operation, $[31,32]$.

2. An operation $f$ is a polymorphism of all relations representable by linear equations over a field $F$ if and only if $f=\alpha_{1} x_{1}+\ldots+\alpha_{n} x_{n}$ where $\alpha_{1}, \ldots, \alpha_{n} \in F$ are such that $\alpha_{1}+\ldots+\alpha_{n}=1,[40]$.

3. If $\mathcal{H}=K_{\ell}$, a complete graph on $\ell>2$ vertices, then an operation $f$ is a polymorphism of $\mathcal{H}$ if and only if $f$ is an essentially unary surjective operation. If $\mathcal{H}=K_{2}$ then $\mathcal{H}$ has a majority polymorphism. 
The examples above and Theorem 1 provide necessary conditions for tractability and expressibility in Datalog.

\section{Corollary 1.}

1. If every polymorphism of a structure $\mathcal{H}$ levery term operation of an algebra $\mathbb{A}]$ is an essentially unary surjective operation then $\operatorname{CSP}(\mathcal{H})[\operatorname{CSP}(\mathbb{A})$, respectively] is NP-complete.

2. If there is a field $F$ such that every polymorphism of a structure $\mathcal{H}$ [every term operation of an algebra $\mathbb{A}]$ is of the form $f=\alpha_{1} x_{1}+\ldots+\alpha_{n} x_{n}$, where $\alpha_{1}, \ldots, \alpha_{n} \in F$ are such that $\alpha_{1}+\ldots+\alpha_{n}=1$, then $\operatorname{CSP}(\mathcal{H})[\operatorname{CSP}(\mathbb{A})$, respectively] is not expressible in Datalog [is not of bounded relational width].

If every term operation of a finite algebra $\mathbb{A}$ is essentially unary surjective then $\mathbb{A}$ is said to be a $G$-set. If there is a module $M$ over a ring $R$ such that every term operation of $\mathbb{A}$ can be represented as $\alpha_{1} x_{1}+\ldots+\alpha_{n} x_{n}$ for $\alpha_{1}, \ldots, \alpha_{n} \in R$ and $\alpha_{1}+\ldots+\alpha_{n}=1$, then $\mathbb{A}$ is called an idempotent reduct of a module.

Example 4 allows one to classify 2-element algebras in terms of complexity.

Proposition 1 (Schaefer's Dichotomy Theorem, [39]). For any 2-element algebra $\mathbb{A}$, the problem $\operatorname{CSP}(\mathbb{A})$ is (globally) tractable if and only if $\operatorname{Term}(\mathbb{A})$ contains one of the following:

- the constant 0 or constant 1 operation;

- the conjunction or disjunction operations (which are semilattice);

- the majority operation $(x \vee y) \wedge(y \vee z) \wedge(z \vee x)$;

- the Mal'tsev operation $x-y+z(\bmod 2)$.

In all other cases $\operatorname{CSP}(\mathbb{A})$ is NP-complete.

\subsection{Varieties}

For the purposes of settling the Dichotomy Conjecture and related questions, the class of algebras to be considered can be significantly reduced. An algebra $\mathbb{A}$ is called surjective if every one of its unary term operations is surjective. One way to transform an algebra into a surjective algebra is as follows: Let $g$ be a unary term operation of $\mathbb{A}=(A ; F)$ with a minimal range. Then $g(\mathbb{A})$ denotes the algebra $\left(g(A) ; F_{g}\right)$ where $F_{g}=\left\{\left.g f\right|_{g(A)} \mid f \in \operatorname{Term}(\mathbb{A})\right\}$. It is not difficult to see that this algebra is surjective. The algebra $\mathbb{A}$ is called idempotent if every one of its term operations $f$ satisfies the equation $f(x, \ldots, x) \approx x$. The full idempotent reduct of $\mathbb{A}$ is the algebra $\operatorname{Id}(\mathbb{A})=\left(A ; F_{i d}\right)$ where $F_{i d}$ is the set of all idempotent operations from $\operatorname{Term}(\mathbb{A})$.

Theorem 2 ([14]). Let $\mathbb{A}$ be an algebra.

1. If $g$ is a unary term operation of $\mathbb{A}$ with minimal range then $\mathbb{A}$ is tractable if and only if $g(\mathbb{A})$ is tractable. The algebra $\mathbb{A}$ is $N P$-complete if and only if $g(\mathbb{A})$ is NP-complete. 
2. If $\mathbb{A}$ is surjective then $\mathbb{A}$ is tractable if and only if $\operatorname{ld}(\mathbb{A})$ is tractable. The algebra $\mathbb{A}$ is $N P$-complete if and only if $\operatorname{ld}(\mathbb{A})$ is NP-complete.

The main idea of the algebraic approach is to use some properties of an algebra in order to determine the complexity of the associated constraint satisfaction problem. To identify these properties, some connections between the complexity of an algebra and the complexity of those algebras that can be obtained from it by some standard algebraic constructions will be very helpful.

- Let $\mathbb{A}=(A ; F)$ be an algebra. The $k$-th direct power of $\mathbb{A}$ is the algebra $\mathbb{A}^{k}=\left(A^{k} ; F\right)$ where we treat each $\left(n\right.$-ary) operation $f \in F$ as acting on $A^{k}$ component-wise.

- Let $\mathbb{A}=(A ; F)$ be an algebra, and let $B$ be a subset of $A$ such that, for any (n-ary) $f \in F$, and for any $b_{1}, \ldots, b_{n} \in B$, we have $f\left(b_{1}, \ldots, b_{n}\right) \in B$. Such a subset is called a subuniverse of $\mathbb{A}$. When $B$ is non-empty, the algebra $\mathbb{B}=\left(B ;\left.F\right|_{B}\right)$, where $\left.F\right|_{B}$ consists of restrictions of operations $f \in F$ to $B$, is called a subalgebra of $\mathbb{A}$.

- Let $\mathbb{A}_{1}=\left(A_{1} ; F_{1}\right)$ and $\mathbb{A}_{2}=\left(A_{2} ; F_{2}\right)$ such that $F_{1}=\left\{f_{i}^{1} \mid i \in I\right\}$, $F_{2}=\left\{f_{i}^{2} \mid i \in I\right\}$, and $f_{i}^{1}, f_{i}^{2}$ are of the same arity, for some set $I$ and each $i \in I$. A mapping $\varphi: A_{1} \rightarrow A_{2}$ is called a homomorphism from $\mathbb{A}_{1}$ to $\mathbb{A}_{2}$ if $\varphi f_{i}^{1}\left(a_{1}, \ldots, a_{n_{i}}\right)=f_{i}^{2}\left(\varphi\left(a_{1}\right), \ldots, \varphi\left(a_{n_{i}}\right)\right)$ holds for all $i \in I$ and all $a_{1}, \ldots, a_{n_{i}} \in A_{1}$. If the mapping $\varphi$ is onto then $\mathbb{A}_{2}$ is said to be a homomorphic image of $\mathbb{A}_{1}$.

By a classic result of Birkhoff (see Theorem 11.9 from [17]), properties of algebras that are preserved under the taking of subalgebras, homomorphic images, and direct products (a natural generalization of the direct power construction) are precisely those that can be defined by equations. We note that except for the last one, all of the properties of the operations listed in Example 4 are defined by equations. Equationally defined classes of algebras, also known as varieties of algebras, are fundamental objects of study in universal algebra $[27,37]$. The following theorems thus provide an important link between the constraint satisfaction problem and universal algebra.

Theorem $3([\mathbf{1 4}, \mathbf{8}])$. Let $\mathbb{A}$ be a finite algebra. Then

1. if $\mathbb{A}$ is tractable then so is every subalgebra, homomorphic image, and direct power of $\mathbb{A}$.

2. if $\mathbb{A}$ has an NP-complete subalgebra, homomorphic image, or direct power, then $\mathbb{A}$ is NP-complete itself.

Theorem 4 ([35]). Let $\mathbb{A}$ be a finite algebra. If $\mathbb{A}$ has bounded width then every subalgebra, homomorphic image, and direct power of $\mathbb{A}$ has bounded width.

Using Birkhoff's Theorem, the variety that an algebra $\mathbb{A}$ determines, denoted by $\operatorname{var}(\mathbb{A})$, can be defined either as the class of all algebras that satisfy the same equations that $\mathbb{A}$ does, or as the class of all algebras that arise as homomorphic images of subalgebras of direct powers of $\mathbb{A}$. 


\section{Corollary 2.}

1. If $\mathbb{A}$ is tractable then so is every finite algebra from $\operatorname{var}(\mathbb{A})$. If $\operatorname{var}(\mathbb{A})$ contains an NP-complete algebra then $\mathbb{A}$ is NP-complete.

2. If $\mathbb{A}$ has bounded width then every finite algebra from $\operatorname{var}(\mathbb{A})$ has bounded width. If $\operatorname{var}(\mathbb{A})$ contains an algebra of unbounded width then $\mathbb{A}$ does not have bounded width.

3. Tractability, NP-completeness, and bounded width are properties of an algebra that depend only on the equations satisfied by the algebra.

Using Corollary 2 we can strengthen Corollary 1 as follows.

Theorem $5([\mathbf{1 4}, \mathbf{3 5}])$. Let $\mathbb{A}$ be an algebra

1. If $\operatorname{var}(\mathbb{A})$ contains a $G$-set then $\mathbb{A}$ is NP-complete.

2. If $\operatorname{var}(\mathbb{A})$ contains a reduct of a module then $\mathbb{A}$ does not have bounded width.

To date no NP-complete or unbounded width algebra is known that does not satisfy the corresponding condition of Theorem 5 . It is widely believed that these necessary conditions are also sufficient, at least for idempotent algebras.

Conjecture 1 (complexity dichotomy conjecture) An idempotent algebra $\mathbb{A}$ is tractable if and only if $\operatorname{var}(\mathbb{A})$ does not contain a $G$-set. Otherwise it is NP-complete.

Conjecture 2 (bounded width conjecture) An idempotent algebra $\mathbb{A}$ has bounded width if and only if $\operatorname{var}(\mathbb{A})$ does not contain a reduct of a module.

Conjectures 1 and 2 have been proved in a number of particular cases: 2element algebras ([39]), 3-element algebras ([12]), semigroups $([9,23])$. The following example shows that the undirected graphs dichotomy theorem by Hell and Nešetřil [26] also fits Conjecture 1.

Example 7 ([11]). Let $H$ be an undirected graph, $\mathbb{A}=A \lg (H)$, and $g$ a unary term operation of $\mathbb{A}$ with a minimal range. Then $H$ is non-bipartite if and only if $\operatorname{var}(g(\mathbb{A}))$ contains a G-set. Otherwise $g(H)$ is $K_{2}$ and $g(\mathbb{A})$ has a majority term operation.

\section{Alternate versions of the conjectures}

The goal of this section is to present new formulations of Conjectures 1 and 2 that have emerged over the past several years. Central to our first formulation is the notion of a congruence of an algebra $\mathbb{A}$. A congruence $\theta$ of $\mathbb{A}$ is an equivalence relation on $A$ that is invariant under all basic (and therefore term) operations of $\mathbb{A}$. Every algebra $\mathbb{A}$ has two distinguished congruences $0_{A}$ and $1_{A}$ corresponding to the smallest and largest equivalence relations on the set $A$. For $\theta$ a congruence of $\mathbb{A}=(A ; F)$ and $a \in A$ by $a / \theta$ we denote the $\theta$-class containing $a$; and denote $\left\{a /_{\theta} \mid a \in A\right\}$, the set of all $\theta$-classes, by $A /_{\theta}$. The quotient algebra $\mathbb{A} /_{\theta}$ is the 
algebra with universe $A / \theta$ and whose basic operations are $\left\{f /_{\theta}: f \in F\right\}$, where for $f \in F$,

$$
f /{ }_{\theta}\left(a_{1} /{ }_{\theta}, \ldots, a_{n} / \theta_{\theta}\right)=\left(f\left(a_{1}, \ldots, a_{n}\right)\right) /_{\theta} .
$$

It is elementary that the mapping $\varphi: \mathbb{A} \rightarrow \mathbb{A} / \theta$ that maps an element $a \in A$ to $a / \theta$ is a surjective homomorphism and so it follows that $\mathbb{A} / \theta$ is a homomorphic image of $\mathbb{A}$.

\subsection{Tame Congruence Theory}

In the early 1980's Hobby and McKenzie developed a theory of the local structure of finite algebras called tame congruence theory [27]. At the heart of the theory is a notion of a neighbourhood of a finite algebra, relativized to certain congruences of the algebra. The local structure of a finite algebra that emerges from their theory is surprisingly well-behaved and has been used to prove many striking theorems in universal algebra.

Definition 1. Let $\mathbb{A}$ be a finite algebra and $\alpha$ a minimal congruence of $\mathbb{A}$ (i.e., $0_{A}<\alpha$ and if $\beta$ is a congruence of $\mathbb{A}$ with $0_{A}<\beta \leq \alpha$ then $\beta=\alpha$.)

- An $\alpha$-minimal set of $\mathbb{A}$ is a subset $U$ of $A$ such that

- $U=p(A)$ for some unary polynomial $p(x)$ of $\mathbb{A}$ that is not constant on at least one $\alpha$-class, and

- with respect to containment, $U$ is minimal with this property.

- An $\alpha$-neighbourhood (or $\alpha$-trace) of $A$ is a subset $N$ of $A$ such that

- $N=U \cap(a / \alpha)$ for some $\alpha$-minimal set $U$ and $\alpha$-class $a / \alpha$, and

- $|N|>1$.

It follows from the definition that a given $\alpha$-minimal set $U$ contains within it at least one (and possibly several) $\alpha$-neighbourhoods. The union of all of the $\alpha$-neighbourhoods in $U$ is called the body of $U$, while the remaining elements of $U$ form the tail of $U$. What is surprising is that the structure that $\mathbb{A}$ induces on any one of its $\alpha$-neighbourhoods is quite uniform and is restricted to one of five possible types. What is meant by induced structure is given in the next definition.

Definition 2. Let $\mathbb{A}$ be an algebra and $U \subseteq A$. The algebra induced by $\mathbb{A}$ on $U$ is the algebra with universe $U$ whose basic operations consist of the restriction to $U$ of all polynomials of $\mathbb{A}$ under which $U$ is closed. We denote this induced algebra by $\left.\mathbb{A}\right|_{U}$.

Note the difference between this notion and the more familiar one of subuniverse (recall that a subuniverse of an algebra $\mathbb{A}$ is a subset of $A$ that is closed under all term operations of $\mathbb{A}$ ). In the theory developed by Hobby and McKenzie, the polynomials of an algebra play a central role and in fact, two finite polynomially equivalent algebras (i.e., two algebras over the same universe whose sets of polynomials coincide) are, for the most part, indistinguishable using tame congruence theory. 
Theorem 6. Let $\mathbb{A}$ be a finite algebra and $\alpha$ a minimal congruence of $\mathbb{A}$.

- If $U$ and $V$ are $\alpha$-minimal sets then $\left.\mathbb{A}\right|_{U}$ and $\left.\mathbb{A}\right|_{V}$ are isomorphic and in fact there is a polynomial $p(x)$ of $\mathbb{A}$ that maps $U$ bijectively on to $V$.

- If $N$ and $M$ are $\alpha$-neighbourhoods then $\left.\mathbb{A}\right|_{N}$ and $\left.\mathbb{A}\right|_{M}$ are isomorphic via the restriction of some polynomial of $\mathbb{A}$.

- If $N$ is an $\alpha$-neighbourhood then $\left.\mathbb{A}\right|_{N}$ is polynomially equivalent to one of:

1. A unary algebra whose basic operations are all permutations (unary type);

2. A one-dimensional vector space over some finite field (affine type);

3. A 2-element boolean algebra (boolean type);

4. A 2-element lattice (lattice type);

5. A 2-element semilattice (semilattice type).

Much more can be said about the $\alpha$-neighbourhoods and minimal sets of an algebra but for now we point out that the previous theorem allows us to assign a type to each minimal congruence $\alpha$ of an algebra according to the behaviour of the $\alpha$-neighbourhoods. For example, a minimal congruence whose $\alpha$-neighbourhoods are all polynomially equivalent to a vector-space is said to have affine type (or to have type 2).

In Figure 1 two $\alpha$-minimal sets of an algebra $\mathbb{A}, U$ and $V$, of a minimal congruence $\alpha$ are pictured, along with two $\alpha$-neighbourhoods, $N$ and $M$, contained in them. The dashed lines delineate the $\alpha$-blocks of the algebra.

Taking this idea one step further, given a pair of congruences $(\alpha, \beta)$ of $\mathbb{A}$ with $\beta$ covering $\alpha$ (i.e., $\alpha<\beta$ and there are no congruences of $\mathbb{A}$ strictly between the two), one can form the quotient algebra $\mathbb{A} / \alpha$ and then consider the congruence $\beta / \alpha=\{(a / \alpha, b / \alpha):(a, b) \in \beta\}$. Since $\beta$ covers $\alpha$ in the congruence lattice of $\mathbb{A}$ then $\beta / \alpha$ is a minimal congruence of $\mathbb{A} / \alpha$ and so can be assigned one of the five types. In this way we can assign to each covering pair of congruences of $\mathbb{A}$ a type and so end up with a labelled congruence lattice for $\mathbb{A}$.

For modestly sized algebras, one can, without too much effort, compute their labelled congruence lattices. Since in general, the size of the congruence lattice of a finite algebra can be much larger than the algebra, the task of computing the labelled congruence lattice of an algebra is by no means tractable. If one is just interested in determining the type of a given covering pair of congruences or in the set of labels that appear in the labelled congruence lattice of an algebra, polynomial time algorithms exist (see [3]).

Example 8. Consider the algebra $\mathbb{A}$ with universe $\{0,1,2,3\}$ having a single binary basic operation $x \cdot y$ defined by:

$$
\begin{array}{c|cccc}
. & 0 & 1 & 2 & 3 \\
\hline 0 & 0 & 0 & 0 & 3 \\
1 & 0 & 1 & 0 & 1 \\
2 & 0 & 0 & 2 & 3 \\
3 & 3 & 1 & 3 & 3
\end{array}
$$




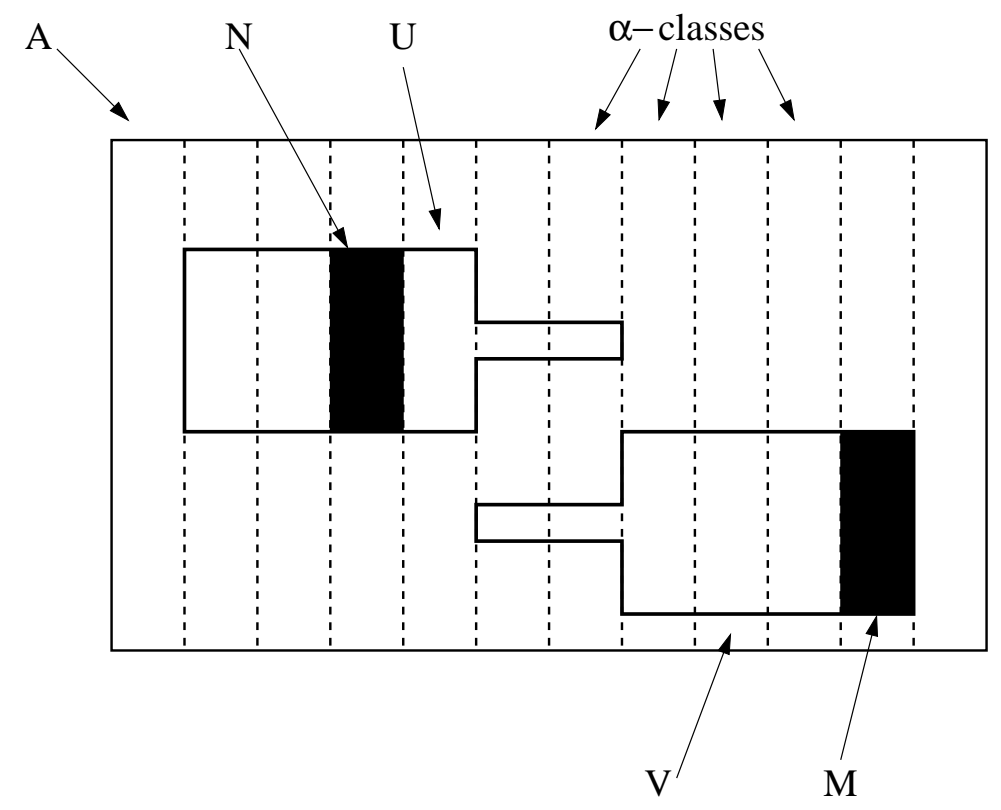

Fig. 1. Minimal Sets

Besides the two congruence $0_{A}$ and $1_{A}, \mathbb{A}$ only has two other (minimal) congruences, $\alpha$ and $\beta$, pictured in Figure 2 as partitions (using the dotted lines) of the universe of $\mathbb{A}$.
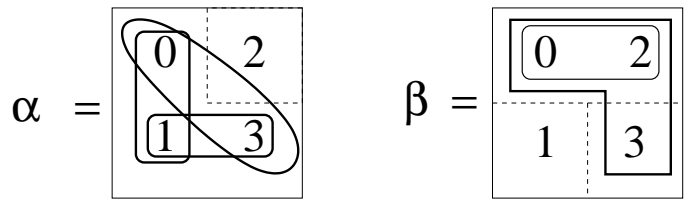

Fig. 2. The Congruences $\alpha$ and $\beta$, with their minimal sets

We claim that the type of $\alpha$ is boolean and the type of $\beta$ is semilattice. To see this, consider the polynomials $p(x)=x \cdot 1$ and $q(x)=x \cdot 2$. The range of $p$ is $\{0,1\}$ and so $N=\{0,1\}$ is both an $\alpha$-minimal set and an $\alpha$-neighbourhood (since $p$ is non constant on some $\alpha$-class and has minimal range subject to this property). On the other hand, the range of $q$ is $\{0,2,3\}$ and so is either a $\beta$-minimal set or properly contains one since $q$ is not constant on the only non- 
trivial $\beta$-class. By analyzing the set of unary polynomials of $\mathbb{A}$ it can be seen that in fact $V=\{0,2,3\}$ is indeed a $\beta$-minimal set and hence that $M=\{0,2\}$ is a $\beta$-neighbourhood.

Now that $\alpha$ and $\beta$-neighbourhoods have been identified, we need only determine the types of the algebras that $\mathbb{A}$ induces on each of them to determine the types of $\alpha$ and $\beta$. We see that the restriction of $x \cdot y$ to $N$ provides a semilattice operation on $N$ and so the type of $\alpha$ cannot be unary or affine since algebras of these types do not support a semilattice polynomial. Since all boolean operations can be obtained by composition from a boolean semilattice operation and complementation, it suffices to produce a unary polynomial of $\mathbb{A}$ that maps 0 to 1 and 1 to 0 in order to establish that the type of $\alpha$ is boolean. It can be checked that the polynomial $(((x \cdot 3) \cdot 2) \cdot 1)$ fits the bill. We leave the details of the calculation of the type of $\beta$ to the reader and conclude the presentation of this example by claiming that the types of the covering pairs $\left(\beta, 1_{A}\right)$ and $\left(\alpha, 1_{A}\right)$ are boolean and semilattice, respectively.

While the type-labelled congruence lattice of a finite algebra carries much information about the algebra, it turns out that just knowing the set of labels that appear in the labelled congruence lattice of a finite algebra or the variety that it generates is useful.

\section{Definition 3.}

1. The typeset of a finite algebra $\mathbb{A}$, denoted typ $\{\mathbb{A}\}$, is the set of labels that appear in its labelled congruence lattice, and so is a subset of $\{$ unary, affine, boolean, lattice, semi-lattice\}.

2. The typeset of a class of algebras $\mathcal{K}$ is the union of the typesets of all of its finite members and is denoted by $\operatorname{typ}\{\mathcal{K}\}$.

3. We say that a finite algebra or a class of algebras omits a particular type if that type does not appear in its typeset.

The following result, found in [8] provides a connection with Conjecture 1, the Complexity Dichotomy Conjecture.

Theorem 7. Let $\mathbb{A}$ be a finite idempotent algebra and $\mathcal{V}$ the variety generated by $\mathbb{A}$. Then $\mathcal{V}$ omits the unary type if and only if $\operatorname{var}(\mathbb{A})$ does not contain a $G$-set. In fact, this condition holds if and only if there is no algebra in $\mathrm{HS}(\mathbb{A})$ that is term equivalent to a set (i.e., whose basic operations are just projections).

This theorem allows us to restate the Complexity Dichotomy Conjecture in terms of types:

Conjecture 1 (the complexity dichotomy conjecture, version 2) A finite idempotent algebra $\mathbb{A}$ is tractable if and only if the variety generated by $\mathbb{A}$ omits the unary type (or equivalently, that every subalgebra of $\mathbb{A}$ omits the unary type).

Something similar occurs when considering Conjecture 2, the Bounded Width Conjecture, namely we can express it in terms of omitting tame congruence theoretic types. 
Theorem 8 ([41]). Let $\mathbb{A}$ be a finite idempotent algebra and $\mathcal{V}$ the variety generated by $\mathbb{A}$. Then $\mathcal{V}$ omits the unary and affine types if and only if $\operatorname{var}(\mathbb{A})$ does not contain an algebra that is term equivalent to a reduct of a module over some finite ring. In fact, this condition holds if and only if there is no algebra in $\mathrm{HS}(\mathbb{A})$ that is term equivalent to a set or to the full idempotent reduct of a module over some finite ring.

In the language of tame congruence theory, the Bounded Width Conjecture becomes:

Conjecture 2 (the bounded width conjecture, version 2) A finite idempotent algebra $\mathbb{A}$ has bounded width if and only if the variety generated by $\mathbb{A}$ omits the unary and affine types (or equivalently, that every subalgebra of $\mathbb{A}$ omits these types).

We conclude this sub-section with a brief discussion of the complexity of determining if a given finite relational structure or finite algebra is tractable or has bounded width, assuming that Conjectures 1 and 2 are true.

Theorem $9([\mathbf{8}, \mathbf{1 5}, \mathbf{4 1}])$. Under the assumption that Conjectures 1 and 2 are true,

1. the problems of determining if a finite relational structure $\mathcal{H}$ is tractable or has bounded width is NP-hard, and

2. the problems of determining if a finite idempotent algebra $\mathbb{A}$ is tractable or has bounded width are polynomial-time solvable.

We note that without the assumption of idempotency, Freese and Valeriote have shown [25] that to determine if the variety generated by a finite algebra omits the unary type or both the unary and affine types are both EXPTIMEcomplete problems.

\subsection{Weak Near-unanimity operations}

Recall that a near-unanimity operation on a set $A$ is a function $t\left(x_{1}, \ldots, x_{n}\right)$, for $n>1$, that satisfies the equations

$$
t(y, x, x, \ldots, x) \approx t(x, y, x, \ldots, x) \approx \cdots \approx t(x, x, \ldots, x, y) \approx x
$$

From [30] we know that if a relational structure $\mathcal{H}$ has a near-unanimity polymorphism then $\operatorname{CSP}(\mathcal{H})$ is tractable. The following variation of this notion was developed by E. Kiss and Valeriote while investigating the Bounded Width Conjecture.

Definition 4. An operation $t\left(x_{1}, \ldots, x_{n}\right)$, for $n>1$, on a set $A$ is a weak nearunanimity operation if it is idempotent and satisfies the equations

$$
t(y, x, x, \ldots, x) \approx t(x, y, x, \ldots, x) \approx \cdots \approx t(x, x, \ldots, x, y)
$$


Clearly any near-unanimity operation is also a weak near-unanimity operation but there are algebras that have term operations of the latter sort but not of the former. For example, for any positive integer $n$, the term operation $x_{1}+x_{2}+$ $\cdots+x_{n+1}$ of the group of integers modulo $n$ is a weak near-unanimity operation. It is not difficult to show that this group fails to have a near-unanimity term operation in any number of variables. We leave it as an exercise to show that the operation $x \cdot(y \cdot z)$ on our 4-element example is a weak near-unanimity operation (and that this algebra does not have a near unanimity term operation).

While it is not too difficult to show that if a finite algebra has a weak nearunanimity term operation then the variety that it generates must omit the unary type, the converse is much more difficult to show. A recent result of Maroti and McKenzie establishes this, along with a characterization of finite algebras that generate varieties that omit both the unary and affine types.

Theorem 10 ([38]). Let $\mathbb{A}$ be a finite algebra and $\mathcal{V}$ the variety that it generates.

1. $\mathcal{V}$ omits the unary type if and only if $\mathbb{A}$ has a weak near-unanimity term operation.

2. $\mathcal{V}$ omits the unary and affine types if and only if there is some $N>0$ such that for all $k \geq N, \mathbb{A}$ has a weak near unanimity term of arity $k$.

This surprising result allows us to provide restatements of the conjectures.

Conjecture 1 (the complexity dichotomy conjecture, version 3) A finite idempotent algebra $\mathbb{A}$ is tractable if and only if $\mathbb{A}$ has a weak near-unanimity term.

Conjecture 2 (the bounded width conjecture, version 3) A finite idempotent algebra $\mathbb{A}$ has bounded width if and only if for all but finitely many $k>0$, $\mathbb{A}$ has a $k$-ary weak near unanimity term.

\section{Tractability via few subpowers}

In this section we discuss a thread of tractability results that culminates in a theorem that unifies them in terms of a notion of a finite algebra having few subpowers.

Definition $5([2])$. A finite algebra $\mathbb{A}$ is said to have few subpowers if there is some polynomial $p(n)$ such that for each $n>0$,

$$
s_{\mathbb{A}}(n)=\log _{2} \mid\left\{B: B \text { is a subuniverse of } \mathbb{A}^{n}\right\} \mid \leq p(n) .
$$

It is not difficult to see that for any finite algebra $\mathbb{A}$ of size $m$, the function $s_{\mathbb{A}}(n)$ is bounded above by $m^{n}$. In general $s_{\mathbb{A}}(n)$ will grow exponentially and so the few subpowers condition imposes certain restrictions on the algebra $\mathbb{A}$. One consequence of a finite algebra $\mathbb{A}$ having few subpowers is the existence of a 
polynomial $g(n)$ such that for any $n>0$, every subalgebra of $\mathbb{A}^{n}$ has a generating set of size bounded above by $g(n)$. In fact this "small generating set" property is equivalent to having few subpowers. Before characterizing such algebras, we present some examples.

Using a theorem of Baker and Pixley from [4] it follows that if $\mathbb{A}$ is a finite algebra that has a $k$-ary near unanimity term operation (see Example 4 ) then the function $s_{\mathbb{A}}(n)$ is bounded above by a polynomial of degree $k-1$ and so such algebras have few subpowers. An early tractability result of Jeavons, Cohen and Cooper [30] establishes that algebras having near unanimity terms are tractable, and it is no coincidence that this tractability result can be proved using the Baker-Pixley theorem.

In [24], Feder and Vardi prove that if a relational structure $\mathcal{H}$ has a polymorphism of the form $x \cdot y^{-1} \cdot z$ for some group operation $x \cdot y$ on $H$ then $\operatorname{CSP}(\mathcal{H})$ is tractable. Generalizing this, Bulatov [5] proves that if a finite algebra $\mathbb{A}$ has a term $p(x, y, z)$ that satisfies the equations $p(x, x, y) \approx p(y, x, x) \approx=y$ for all $x, y \in A$ then $\mathbb{A}$ is also tractable (any operation that satisfies these equations is known as a Mal'tsev operation, see Example 4). The proof of this theorem found in [13] exploits the fact that any finite algebra with a Mal'tsev term has the small generating sets property (and hence, few subpowers).

While Mal'tsev and near unanimity operations are of quite different character, Dalmau in [22] managed to find a common generalization of them via the generalized majority-minority operation (see Example 4 for the definition). In a modification of the algorithm presented in [13], Dalmau shows in [22] that any finite algebra that has a GMM term is tractable. As in the case of algebras with Mal'tsev terms, these algebras have few subpowers and the small generating sets property and it is this latter property that plays a crucial role in the proof.

In [2] a characterization of finite algebras with few subpowers is given in terms of the presence of a special type of operation.

Definition 6. $A k$-edge operation on a set $A$ is a $k+1$-variable operation $t$ that satisfies the equations:

$$
\begin{aligned}
t(x, x, y, y, y, \ldots, y, y) & \approx y \\
t(x, y, x, y, y, \ldots, y, y) & \approx y \\
t(y, y, y, x, y, \ldots, y, y) & \approx y \\
t(y, y, y, y, x, \ldots, y, y) & \approx y \\
\vdots & \\
t(y, y, y, y, y, \ldots, y, x) & \approx y .
\end{aligned}
$$

Theorem 11 ([2]). A finite algebra $\mathbb{A}$ has few subpowers if and only if it has a $k$-edge term for some $k>0$. If this condition fails to hold then the function $s_{\AA}(n)$ grows exponentially.

Using this characterization the tractability of algebras with few subpowers can be deduced. 
Corollary 3 ([28]). If the finite algebra $\mathbb{A}$ has few subpowers then it is globally tractable.

We note that the proof of this corollary closely follows the GMM tractability proof of Dalmau. We also note that the theorem and corollary settle conjectures posed by Chen [18] and Dalmau [21] on the nature of algebras with few subpowers.

We conclude this section with a result of Marković and McKenzie [36,2] that highlights the singular position that algebras with near unanimity term operations occupy. We have already noted that if a finite idempotent algebra has a near unanimity operation, then it has bounded width and few subpowers and so can be shown to be tractable in two distinct ways. The following theorem provides a converse to this.

Theorem 12. Let $\mathbb{A}$ be a finite idempotent algebra. If $\mathbb{A}$ is of bounded width and has few subpowers then it has a near unanimity term operation.

\section{Coloured graphs and finite algebras}

The conditions of tractability and bounded width that appear in Conjectures 1 and 2 are known to be necessary. In order to prove that they are also sufficient for the complexity dichotomy conjecture one needs to design an algorithm (or algorithms) that solves CSPs satisfying the tractability condition, and for the bounded width conjecture, that the constraint propagation algorithm solves CSPs satisfying the bounded width condition. In all known cases algorithms (of proofs of the soundness of algorithms) use some local structure of algebras. Usually this structure can be explained in terms of the action of term operations of algebras on small subsets. In this section we propose an approach to the local structure of a finite idempotent algebra that is based on term operations of the algebra.

\subsection{Coloured graphs of algebras}

\subsection{The graph}

The results of this section were first presented in [10]. We relate to every idempotent finite algebra $\mathbb{A}$ an edge-coloured graph $\operatorname{Gr}(\mathbb{A})$. If $\mathbb{A}=(A ; F)$ and $B \subseteq A$, then by $\langle B\rangle$ we denote the subalgebra generated by $B$, that is the smallest subalgebra of $\mathbb{A}$ containing $B$.

Definition 7. Let $\mathbb{A}=(A ; F)$ be a finite idempotent algebra. The vertex set of the graph $\operatorname{Gr}(\mathbb{A})$ is the universe $A$ of $\mathbb{A}$. A pair ab of vertices is an edge if and only if there exists a congruence $\theta$ of $\langle a, b\rangle$ and a term operation $f$ of $\mathbb{A}$ such that either $f / \theta$ is an affine operation on $\langle a, b\rangle / \theta$, or $f / \theta$ is a semilattice operation on $\{a / \theta, b / \theta\}$, or $f / \theta$ is a majority operation on $\{a / \theta, b / \theta\}$ (see Figure 3).

The color of an edge is defined as follows. 


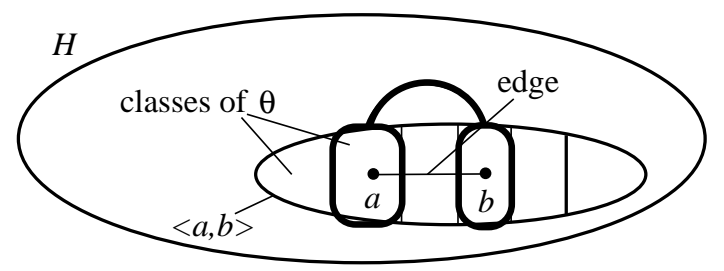

Fig. 3. Edges

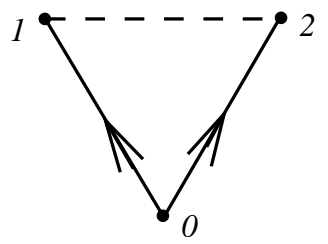

Fig. 4. $\operatorname{Gr}(\mathbb{A})$; edges of the semilattice type are drawn solid, edges of the majority type are dotted

- If there exists a congruence $\theta$ and a term operation $f \in \operatorname{Term}(\mathbb{A})$ such that $\mathrm{f} /{ }_{\theta}$ is a semilattice operation on $\left\{a / \theta, b /{ }_{\theta}\right\}$ then $a b$ is said to have the semilattice type.

- An edge ab is of the majority type if it is not of the majority type and there are a congruence $\theta$ and a term operation $f$ of $\mathbb{A}$ such that $f / \theta$ is a majority operation on $\{a / \theta, b / \theta\}$.

- An edge ab is of the affine type if it is not of the semilattice or majority type and there are a congruence $\theta$ and a term operation $f$ of $\mathbb{A}$ such that $f / \theta$ is an affine operation on $\langle a, b\rangle /_{\theta}$.

We sometimes call the set $a / \theta \cup b / \theta$ a thick edge.

Example 9. Let $\mathbb{A}=(\{0,1,2\} ; f)$ be an algebra, where the operation $f$ is defined by its Cayley table

$$
\begin{array}{rl|lll}
f(x, y) & 0 & 1 & 2 \\
\hline 0 & 0 & 1 & 2 \\
1 & 1 & 1 & 0 \\
2 & 2 & 0 & 2
\end{array}
$$

(In fact, $f$ occurs in [12]; in that paper it is called operation (6).) We have: $\langle 0,1\rangle=\{0,1\},\langle 0,2\rangle=\{0,2\},\langle 1,2\rangle=\{1,2,3\}$; the only congruence of $\langle 1,2\rangle$ such that 1,2 belong to distinct classes is the equality relation; $f$ witnesses that 01 and 02 are edges of semilattice type; 12 cannot be an edge of the semilattice type because no term operation of $\mathbb{A}$ is semilattice on $\{1,2\}$; however, the operation $g(x, y, z)=f(f(x, f(y, z)), f(f(x, y), z))$ is a majority operation on $\{1,2\}$. Thus, $\operatorname{Gr}(\mathbb{A})$ is the graph shown in Figure 4. Note also that this graph was implicitly used in [12] to prove the tractability of $\mathbb{A}$. 
Observe that it is possible that for some pair $a, b$ different congruences of $\langle a, b\rangle$ witness different types of the edge $a b$. Following the definition we always choose the 'strongest' type of the edge. Thus, the semilattice type is stronger than the majority type, which, in turn, is stronger than the affine type.

Example 10. Let $\mathbb{A}, \mathbb{B}$ be algebras with universes $\{0,1\}$ and $\{a, b\}$, respectively, and operations $f, g$. These operations are defined as follows:

- $f$ is a semilattice operation on $\mathbb{A}$, i.e. $f(0,0)=f(0,1)=f(1,0)=0, f(1,1)=$ 1 ;

- $f$ is the first projection on $\mathbb{B}$, i.e. $f(x, y)=x$ for all $x, y \in\{a, b\}$;

$-g$ is the ternary first projection on $\mathbb{A}$, i.e. $g(x, y, z)=x$ for all $x, y, z \in\{0,1\}$;

- $g$ is a majority operation on $\mathbb{B}$; note that there is only one majority operation on a 2-element set.

Then let $\mathbb{C}$ denote the direct product of $\mathbb{A}$ and $\mathbb{B}$, that is the algebra with universe $C=\{(x, y) \mid x \in\{0,1\}, y \in\{a, b\}\}$, and operations $f, g$ on $C$ acting as follows:

$$
f\left(\left(x_{1}, y_{1}\right),\left(x_{2}, y_{2}\right)\right)=\left(f\left(x_{1}, x_{2}\right), f\left(y_{1}, y_{2}\right)\right)
$$

and

$$
g\left(\left(x_{1}, y_{1}\right),\left(x_{2}, y_{2}\right),\left(x_{3}, y_{3}\right)\right)=\left(g\left(x_{1}, x_{2}, x_{3}\right), g\left(y_{1}, y_{2}, y_{3}\right)\right) .
$$

As is easily seen, $\langle(0, a),(1, b)\rangle=\mathbb{C}$ and the equivalence relations $\eta_{1}, \eta_{2}$ defined by $\left(\left(x_{1}, y_{1}\right),\left(x_{2}, y_{2}\right)\right) \in \eta_{1}$ if and only if $x_{1}=x_{2}$, and $\left(\left(x_{1}, y_{1}\right),\left(x_{2}, y_{2}\right)\right) \in \eta_{2}$ if and only if $y_{1}=y_{2}$, are congruences of $\mathbb{C}$. Observe that $f / \eta_{1}$ is a semilattice operation on $\mathbb{C} / \eta_{1}=\left\{(0, a) / \eta_{1},(1, b) / \eta_{1}\right\}$; and that $\mathbb{C} / \eta_{2}=\left\{(0, a) / \eta_{2},(1, b) / \eta_{2}\right\}$ is isomorphic to $\mathbb{B}$. Thus, congruence $\eta_{1}$ witnesses that $(0, a)(1, b)$ is an edge of semilattice type, while $\eta_{2}$ witnesses that the same edge has majority type. Since the semilattice type is stronger, this edge has semilattice type.

\subsection{Connectedness and omitting types}

We show that connectedness of the graph $\operatorname{Gr}(\mathbb{A})$ and the colours of edges that appear in it are closely related to omitting types in the sense of tame congruence theory, and to Conjectures 1 and 2.

Theorem 13 ([10]). For an idempotent algebra $\mathbb{A}$ the following conditions are equivalent:

(1) $\operatorname{var}(\mathbb{A})$ omits the unary type;

(2) $\operatorname{var}(\mathbb{A})$ does not contain a G-set;

(3) for any subalgebra $\mathbb{B}$ of $\mathbb{A}$ the graph $\operatorname{Gr}(\mathbb{B})$ is connected.

We shall refer to condition (3) from Theorem 13 as to the connectedness condition.

Theorem 14. Let $\mathbb{A}$ be an idempotent algebra. The following conditions are equivalent: 
(1) $\operatorname{var}(\mathbb{A})$ omits the unary and affine types;

(2) $\operatorname{var}(\mathbb{A})$ does not contain an algebra that is term equivalent to a reduct of a module over some finite ring;

(3) $\mathbb{A}$ satisfies the connectedness condition, and $\operatorname{Gr}(\mathbb{A})$ does not contain edges of the affine type.

Since this result appears here for the first time we give a proof of it. We shall use an improved version of Lemma 1 from [10].

Lemma 1. Let $\mathbb{A}$ be a finite idempotent algebra, and let $a b$ be an edge of the affine type in $\operatorname{Gr}(\mathbb{A})$. Then there are a maximal congruence $\theta$ of $\langle a, b\rangle$ (that is there is no congruence strictly between $\theta$ and the total congruence) and a module $M$ with the universe $\langle a, b\rangle /{ }_{\theta}$ over a ring $R$ such that every term operation of $\langle a, b\rangle /_{\theta}$ can be represented as an operation $\alpha_{1} x_{1}+\ldots+\alpha_{n} x_{n}$ of $M$ with $\alpha_{1}, \ldots, \alpha_{n} \in R, \alpha_{1}+\ldots+\alpha_{n}=1$.

Proof (of Theorem 14): The equivalence of (1) and (2) is follows from Theorem 8 . We show that (3) is equivalent to (1).

If for some subalgebra $\mathbb{B}$ of $\mathbb{A}$ the graph $\operatorname{Gr}(\mathbb{B})$ is not connected then by Theorem $13 \operatorname{var}(\mathbb{B}) \subseteq \operatorname{var}(\mathbb{A})$ contains a G-set that is term equivalent to a reduct of any module, because in an idempotent variety any G-set is term equivalent to an algebra whose basic operations are projections. If $\operatorname{Gr}(\mathbb{A})$ contains an edge of the affine type $a b$ then by Lemma 1 the algebra $\langle a, b\rangle / \theta$ for a certain $\theta$ is a reduct of a module.

By Theorem 8 if $\operatorname{var}(\mathbb{A})$ contains an algebra term equivalent to a reduct of a module, then there is a subalgebra $\mathbb{B}$ of $\mathbb{A}$ and a congruence $\theta$ of $\mathbb{B}$ such that $\mathbb{B} / \theta$ is term equivalent to a reduct of a module. If this algebra is a G-set then $\operatorname{Gr}(\mathbb{B})$ is not connected by Theorem 13 . Otherwise we assume that $\mathbb{B}$ is a minimal (with respect to containment) subalgebra with this property and $\theta$ is a maximal congruence of $\mathbb{B}$. Then $\theta$ is the only maximal congruence of $\mathbb{B}$. Indeed, if $\eta$ is another maximal congruence of $\mathbb{B}$, then any class $C$ of $\eta$ that is not contained in a class of $\theta$ induces a proper subalgebra $\mathbb{C}$ of $\mathbb{B}$, and $\mathbb{C} / \theta$ is still term equivalent to a reduct of a module; a contradiction with minimality of $\mathbb{B}$. It is not hard to see, that, for any $a, b \in \mathbb{B}$ such that $(a, b) \notin \theta$, the pair $a b$ is an edge of the affine type.

Using Theorems 13 and 14 we can give yet another formulation of the complexity and bounded width conjectures.

Conjecture 1 (the complexity dichotomy conjecture, version 4) A finite idempotent algebra is tractable if and only if it satisfies the connectedness condition.

Conjecture 2 (the bounded width conjecture, version 4) A finite idempotent algebra $\mathbb{A}$ has bounded width if and only if it satisfies the connectedness condition and the graph $\operatorname{Gr}(\mathbb{A})$ does not contain edges of the affine type. 


\subsection{Improving an algebra}

The study of finite algebras in the context of the complexity of the CSP does not necessarily suppose investigation of the exact structure of finite algebras. Therefore we can transform algebras under consideration as long as such a transformation preserves properties supposedly responsible for tractability, e.g. omitting the unary type. In this subsection we show two such transformations.

We say that the graph $\operatorname{Gr}(\mathbb{A})$ is semilattice-connected, if for any two vertices $a, b \in \mathbb{A}$ there is a path in $\operatorname{Gr}(\mathbb{A})$ consisting of edges of the semilattice type. The semilattice/majority connectedness of $\operatorname{Gr}(\mathbb{A})$ is defined similarly.

Proposition 2. Let $\mathbb{A}$ be an idempotent algebra satisfying the connectedness condition, let $a b$ be an edge of $\operatorname{Gr}(\mathbb{A})$ of the semilattice or majority type, and let $R_{a b}=\left(a /{ }_{\theta} \cup b / \theta\right)$ be the corresponding thick edge, where $\theta$ is a congruence certifying the type of $a b$.

(1) $\mathbb{A}_{a b}=\left(A ; F^{\prime}\right)$, where $F^{\prime}$ is the set of all term operations of $\mathbb{A}$ preserving $R_{a b}$, satisfies the connectedness condition.

(2) If $a b$ is has the semilattice type and $\operatorname{Gr}(\mathbb{A})$ is semilattice-connected, then $\operatorname{Gr}\left(\mathbb{A}_{a b}\right)$ is semilattice-connected.

(3) If ab has the majority type and $\operatorname{Gr}(\mathbb{A})$ is semilattice/majority-connected, then $\operatorname{Gr}\left(\mathbb{A}_{a b}\right)$ is semilattice/majority-connected.

As the following example shows, constructing a reduct by adding an edge of the affine type can destroy the connectedness condition and even make a tractable algebra NP-complete.

Example 11. Let $\mathbb{A}=(\{0,1,2\} ; h)$, where $h(x, y, z)=x-y+z$ and,+- denote the operation of addition and subtraction modulo 3 . It is well known (see e.g. [40]) that the term operations of $\mathbb{A}$ are the operations of the form $\alpha_{1} x_{1}+\ldots+$ $\alpha_{n} x_{n}$, where $\alpha_{1}, \ldots, \alpha_{n}$ are integers and $\alpha_{1}+\ldots+\alpha_{n}=1(\bmod 3)$. Therefore, for any $a, b \in A,\langle a, b\rangle=A$, the only maximal congruence of $\langle a, b\rangle$ is the equality relation, and $a b$ is an edge of the affine type.

Since the affine operation $x-y+z$ is an operation of $\mathbb{A}$, the problem $\operatorname{CSP}(\mathbb{A})$ can be solved by Gaussian elimination [31]. Take an edge of $\operatorname{Gr}(\mathbb{A})$, say 01 and a term operation $f\left(x_{1}, \ldots, x_{n}\right)=\alpha_{1} x_{1}+\ldots+\alpha_{n} x_{n}$ of $\mathbb{A}$. If $f$ preserves $\{0,1\}$, then, for any $i \in\{1, \ldots, n\}$, we have $f(0, \ldots, 0,1,0, \ldots, 0)=\alpha_{i} \in\{0,1\}$ (1 is on the $i$ th place). Furthermore, if $\alpha_{i}, \alpha_{j}=1$, then $f(0, \ldots, 0,1,0, \ldots, 0,1,0, \ldots, 0)=$ $\alpha_{i}+\alpha_{j}=2 \notin\{0,1\}$ ( $1 \mathrm{~s}$ are on the $i$ th and $j$ th places). Thus, only one of the $\alpha \mathrm{s}$ is non-zero, which means that $f$ is a projection. Hence, every term operation of $\mathbb{A}_{01}$ is a projection and $\operatorname{CSP}\left(\mathbb{A}_{01}\right)$ is NP-complete.

Proposition 2 amounts to saying that we may restrict our attention to algebras $\mathbb{A}$ such that every thick edge of the semilattice or majority type of $\operatorname{Gr}(\mathbb{A})$ is a subalgebra.

The second transformation preserving the connectedness condition is based on the following statement that shows that the term operations certifying the type of edges can be significantly unified. 
Proposition 3. Let $\mathbb{A}$ be an idempotent algebra. For an edge, $\theta$ always denotes a congruence certifying its type. There are term operations $f, g, h$ of $\mathbb{A}$ such that

$\left.f\right|_{\left\{a / \theta_{\theta}, b / \theta_{\theta}\right\}}$ is a semilattice operation if ab is an edge of the semilattice type, it is the first projection if $a b$ is an edge of the majority or affine type;

$\left.g\right|_{\left\{a / \theta_{\theta}, b / \theta^{\}}\right.}$is a majority operation if ab is an edge of the majority type, it is the first projection if $a b$ is an edge of the affine type, and $\left.\right|_{\{a / \theta, b / \theta\}}(x, y, z)=$ $\left.f\right|_{\left\{a / \theta_{\theta}, b / \theta^{\}}\right.}\left(x,\left.f\right|_{\left\{a / \theta^{, b /}\right\}^{\}}}(y, z)\right)$ if ab has the semilattice type;

$h_{\langle a b\rangle / \theta}$ is an affine operation operation if ab is an edge of the affine type, it is the first projection if $a b$ is an edge of the majority type, and $h_{\left\{a / \theta^{\left., b / \theta_{a b}\right\}}\right.}(x, y, z)=$ $\left.f\right|_{\left\{a / \theta^{, b / \theta}\right\}}\left(x,\left.f\right|_{\left\{a / \theta^{, b / \theta}\right\}}(y, z)\right)$ if ab has the semilattice type.

Example 9 (continued). Let us reconsider the algebra $\mathbb{A}$ from Example 9. By Proposition 2 , since 12 is an edge of the majority type, the algebra $\mathbb{A}_{12}$ satisfies the connectedness condition. The operations $f, g, h$ satisfying the conditions of Proposition 3 can be chosen as follows: $g$ is the operation obtained in Example 9, $f(x, y)=g(x, x, y)$ (the binary operation defined in Example 9 does not fit, because it does not preserve $\{1,2\})$ and $h(x, y, z)=f(x, f(y, z))$.

Propositions 2 and 3 together allow us to restrict ourselves to the study of idempotent algebras that have at most three basic operations, one binary and two ternary, and such that, for any edge of the semilattice or majority $a b$ and a congruence $\theta$ certifying this, the thick edge $a / \theta \cup b / \theta$ is a subalgebra. In the next section we shall see that the class of algebras to be studied can be further narrowed down.

Edges of the semilattice type In this section we focus on edges of the semilattice type of the graph $\operatorname{Gr}(\mathbb{A})$. Note first that if one fixes a congruence $\theta_{a b}$ for each edge of $\operatorname{Gr}(\mathbb{A})$ that certifies its type, and a term operation $f$ such that $f$ is a semilattice operation on $\left\{a /_{\theta_{a b}}, b / \theta_{a b}\right\}$ for every edge $a b$ of the semilattice type of $\operatorname{Gr}(\mathbb{A})$, then one can define an orientation of every such edge. An edge $a b$ of the semilattice type is oriented from $a$ to $b$ if $f\left(a / \theta_{a b}, b / \theta_{a b}\right)=f\left(b / \theta_{a b}, a / \theta_{a b}\right)=$ $b / \theta_{a b}$. For instance, the edges 01, 02 of the graph from Example 9 are oriented from 0 to 1 and 2 respectively. Clearly, orientation strongly depends on the choice of the operation $f$. The graph $\operatorname{Gr}(\mathbb{A})$ oriented accordingly to a term operation $f$ will be denoted by $\operatorname{Gr}_{f}(\mathbb{A})$. We then can define semilattice-connected and strongly semilattice-connected components of $\operatorname{Gr}_{f}(\mathbb{A})$. We will also use the natural order on the set of strongly semilattice-connected components of $\mathrm{Gr}_{f}(\mathbb{A})$ : for components $A, B, A \leq B$ if there is a directed path in $\operatorname{Gr}_{f}(\mathbb{A})$ consisting of edges of the semilattice type and connecting a vertex from $A$ with a vertex from $B$. Later we show that certain restrictions on the set of strongly semilattice-connected components of $\operatorname{Gr}_{f}(\mathbb{A})$ yield the tractability of $\operatorname{CSP}(\mathbb{A})$. 
First we show that if for an edge $a b$ of the semilattice type there is no semilattice term operation on the set $\{a, b\}$ then $a b$ can be thrown out of the graph $\operatorname{Gr}(\mathbb{A})$ such that the connectedness condition is preserved in the remaining graph. Therefore, we can assume that for any edge of the majority type $a b$ there is a semilattice term operation on $\{a, b\}$.

Proposition 4. Let $\mathbb{A}$ be an algebra and $\mathrm{Gr}^{\prime}(\mathbb{A})$ the subgraph of $\operatorname{Gr}(\mathbb{A})$ obtained by omitting edges ab of the semilattice type such that there is no semilattice operation on $\{a, b\}$. Then $\mathrm{Gr}^{\prime}(\mathbb{A})$ is connected. If $\operatorname{Gr}(\mathbb{A})$ is semilattice-connected then $\mathrm{Gr}^{\prime}(\mathbb{A})$ is semilattice-connected. If $\mathrm{Gr}(\mathbb{A})$ is semilattice/majority-connected then $\mathrm{Gr}^{\prime}(\mathbb{A})$ is semilattice/majority-connected.

The graph $\operatorname{Gr}^{\prime}(\mathbb{A})$ oriented according to a binary term operation $f$ will be denoted by $\mathrm{Gr}_{f}^{\prime}(\mathbb{A})$.

We conclude this subsection with a result that shows how properties of the graph $\operatorname{Gr}(\mathbb{A})$ can help in establishing the tractability and bounded width of the algebra $\mathbb{A}$. Let us consider algebras $\mathbb{A}$ with a binary term operation $f$ such that, for every subalgebra $\mathbb{B}$ of $\mathbb{A}$, the subgraph of $\operatorname{Gr}_{f}^{\prime}(\mathbb{A})$ induced by $\mathbb{B}$ has a unique maximal strongly semilattice-connected component. This condition we shall call the maximal semilattice component condition.

Theorem 15. If an algebra $\mathbb{A}$ satisfies the maximal semilattice component condition, then $\operatorname{CSP}(\mathbb{A})$ is of relational width 3 .

Observe that a 2-semilattice, that is a groupoid with a 2-semilattice basic operation, satisfies the maximal semilattice component condition. Indeed, if $\mathbb{A}$ has a 2-semilattice term operation $f$, then $f$ is a semilattice operation on $\{a, f(a, b)\}$ and $\{b, f(a, b)=f(b, a)\}$. This means that $\mathrm{Gr}_{f}^{\prime}(\mathbb{A})$ is semilatticeconnected. Moreover, if $a, b$ belong to different maximal strongly semilatticeconnected components $B$ and $C$, then $f(a, b)$ belongs to a strongly semilatticeconnected component $D$ such that $B \leq D$ and $C \leq D$, a contradiction with the maximality of $B, C$. The same argument is valid for any subalgebra of $\mathbb{A}$, thus, $\mathbb{A}$ satisfies the maximal semilattice component condition. Since every semilattice operation is also a 2-semilattice operation, the same holds for algebras with a semilattice term operation. Thus, by Theorem 15, we obtain the main result of [6], and also the results of [7], since a binary commutative conservative operation is a 2 -semilattice operation, and also the results of $[31,29]$ concerning semilattice operations.

\subsection{Conservative algebras and their graphs}

Let $\mathcal{H}$ be a relational structure. In the conservative (list) constraint satisfaction problem, denoted $\operatorname{CCSP}(\mathcal{H})$, the question is, given a structure $\mathcal{G}$ and, for each element $g \in \mathcal{G}$, a list $L(g)$ of elements of $\mathcal{H}$, whether there exists a homomorphism $\varphi: \mathcal{G} \rightarrow \mathcal{H}$ such that $\varphi(g) \in L(g)$ for all $g \in \mathcal{G}$.

Example 12 (List-H-Colouring). Let $H$ be a (directed) graph. In the LisT $H$-Colouring problem we are given a graph $G$ and, for each vertex $v$ of $G$, 
a set $L(v)$ of vertices of $H$. The question is whether there is a homomorphism $\varphi$ from $G$ to $H$ such that $\varphi(v) \in L(v)$ for every vertex $v$ of $G$. Clearly, List $H$-COLOURING can be represented in the form of the conservative CSP.

Notice that, for any structure $\mathcal{H}$, the problem $\operatorname{CCSP}(\mathcal{H})$ is equivalent to $\operatorname{CSP}\left(\mathcal{H}^{*}\right)$, where $\mathcal{H}^{*}$ is an expansion of $\mathcal{H}$ obtained by adding all unary relations. A structure $\mathcal{H}$ such that for each subset $S \subseteq H$ there is a relational symbol $R$ in the vocabulary with $R^{\mathcal{H}}=S$ is said to be conservative. Thus, instead of conservative CSPs we may study ordinary constraint satisfaction problems corresponding to conservative structures.

On the algebraic side, every term operation $f$ of an algebra $\mathbb{A}$ that gives rise to a conservative CSP must be conservative, that is $f\left(x_{1}, \ldots, x_{n}\right) \in\left\{x_{1}, \ldots, x_{n}\right\}$ for all $x_{1}, \ldots, x_{n}$. Algebras satisfying this condition are also called conservative.

If $\mathbb{A}$ is a conservative algebra, then in particular every 2-element subset of $A$ induces a subalgebra of $\mathbb{A}$. Therefore, $\mathbb{A}$ satisfies the connectedness condition if and only if every pair of its elements constitutes an edge of $\operatorname{Gr}(\mathbb{A})$. Moreover, every edge of this graph is 2-element, implying that the operations $f, g, h$ constructed in Proposition 3 are a semilattice (that is conjunction or disjunction) operation, the majority operation $(x \vee y) \wedge(y \vee z) \wedge(z \vee x)$, and the Mal'tsev operation $x-y+z(\bmod 2)$ on each 2 -element subset from $\mathbb{A}$, respectively (we denote the elements of this subset by 0 and 1 ).

Theorem 16. A conservative algebra $\mathbb{A}$ is tractable if and only if it satisfies the connectedness condition, that is, for any 2-element subalgebra $\mathbb{B}$ of $\mathbb{A}$ (we assume $B=\{0,1\})$, there exists a term operation $t$ such that $\eta_{B}$ is either a semilattice operation $x \vee y$ or $x \wedge y$, or the majority operation $(x \vee y) \wedge(y \vee z) \wedge(z \vee x)$, or the Mal'tsev operation $x-y+z(\bmod 2)$. In this case $\mathbb{A}$ is also globally tractable. Otherwise $\mathbb{A}$ is NP-complete.

Observe that by Proposition 3 the tractability of a conservative algebra is witnessed by term operations of arity at most 3 . This observation implies a stronger version of Theorem 16. An algebra such that each of its $k$-element subsets induces a subalgebra is called $k$-conservative.

Corollary 4. If $\mathbb{A}$ is a 3-conservative algebra then $\mathbb{A}$ is globally tractable if and only if it satisfies the connectedness condition. Otherwise it is NP-complete.

\section{References}

1. Eric Allender, Michael Bauland, Neil Immerman, Henning Schnoor, and Heribert Vollmer. The complexity of satisfiability problems: Refining schaefer's theorem. In MFCS, pages 71-82, 2005.

2. Joel Berman, Paweł Idziak, Petar Marković, Ralph McKenzie, Matthew Valeriote, and Ross Willard. Varieties with few subalgebras of powers. preprint, 2006.

3. Joel D. Berman, Emil W. Kiss, Péter Pröhle, and Ágnes Szendrei. The set of types of a finitely generated variety. Discrete Math., 112(1-3):1-20, 1993.

4. K.A. Baker and A.F. Pixley. Polynomial interpolation and the chinese remainder theorem. Mathematische Zeitschrift, 143:165-174, 1975. 
5. A.A. Bulatov. Mal'tsev constraints are tractable. Technical Report PRG-RR-0205, Computing Laboratory, University of Oxford, Oxford, UK, 2002.

6. A.A. Bulatov. Combinatorial problems raised from 2-semilattices. Journal of Algebra, 298(2):321-339, 2006.

7. A.A. Bulatov and P.G. Jeavons. Tractable constraints closed under a binary operation. Technical Report PRG-TR-12-00, Computing Laboratory, University of Oxford, Oxford, UK, 2000.

8. A.A. Bulatov and P.G. Jeavons. Algebraic structures in combinatorial problems. Technical Report MATH-AL-4-2001, Technische universität Dresden, Dresden, Germany, 2001. available at http://web.comlab.ox.ac.uk/oucl/research/areas/ constraints/publications/index.html.

9. A.A. Bulatov, P.G. Jeavons, and M.V. Volkov. Finite semigroups imposing tractable constraints. In Gracinda M.S.Gomes, Jean-Eric Pin, Pedro V.Silva, editor, Semigroups, Algorithms, Automata and Languages, pages 313-329. World Scientific, Singapore, 2002.

10. Andrei A. Bulatov. A graph of a relational structure and constraint satisfaction problems. In $L I C S$, pages 448-457, 2004.

11. Andrei A. Bulatov. H-coloring dichotomy revisited. Theor. Comput. Sci., 349(1):31-39, 2005.

12. Andrei A. Bulatov. A dichotomy theorem for constraint satisfaction problems on a 3-element set. J. ACM, 53(1):66-120, 2006.

13. Andrei A. Bulatov and Víctor Dalmau. A simple algorithm for mal'tsev constraints. SIAM J. Comput., 36(1):16-27, 2006.

14. Andrei A. Bulatov, Peter Jeavons, and Andrei A. Krokhin. Classifying the complexity of constraints using finite algebras. SIAM J. Comput., 34(3):720-742, 2005.

15. Albert Atserias, Andrei Bulatov, and Anuj Dawar. Affine systems of equations and counting infinitary logic. In Proceedings of the 34th International Colloquium on Automata, Languages and Programming-ICALP'07, 2007.

16. Andrei A. Bulatov, Andrei A. Krokhin, and Benoit Larose. Dualities for Constraint Satisfaction Problems. submitted.

17. S. Burris and H.P. Sankappanavar. A course in universal algebra, volume 78 of Graduate Texts in Mathematics. Springer-Verlag, New York-Berlin, 1981.

18. Hubie Chen. The expressive rate of constraints. Ann. Math. Artif. Intell., 44(4):341-352, 2005.

19. Matthias Clasen and Matthew Valeriote. Tame congruence theory. In Lectures on algebraic model theory, volume 15 of Fields Inst. Monogr., pages 67-111. Amer. Math. Soc., Providence, RI, 2002.

20. V. Dalmau. A new tractable class of constraint satisfaction problems. In Proceedings 6th International Symposium on Artificial Intelligence and Mathematics, 2000.

21. V. Dalmau. Computational Complexity of Problems over Generalised Formulas. PhD thesis, Department LSI of the Universitat Politecnica de Catalunya (UPC), Barcelona., March, 2000.

22. Víctor Dalmau. Generalized majority-minority operations are tractable. In LICS, pages 438-447, 2005.

23. Víctor Dalmau, Ricard Gavaldà, Pascal Tesson, and Denis Thérien. Tractable clones of polynomials over semigroups. In $C P$, pages 196-210, 2005.

24. T. Feder and M.Y. Vardi. The computational structure of monotone monadic SNP and constraint satisfaction: A study through datalog and group theory. SIAM Journal of Computing, 28:57-104, 1998. 
25. Ralph S. Freese and Matthew A. Valeriote. On the complexity of some Maltsev conditions. preprint, 2006.

26. P. Hell and J. Nešetřil. On the complexity of H-coloring. Journal of Combinatorial Theory, Ser.B, 48:92-110, 1990.

27. D. Hobby and R.N. McKenzie. The Structure of Finite Algebras, volume 76 of Contemporary Mathematics. American Mathematical Society, Providence, R.I., 1988.

28. Paweł Idziak, Petar Marković, Ralph McKenzie, Matthew Valeriote, and Ross Willard. Tractability and learnability arising from algebras with few subpowers. In LICS '07: Proceedings of the 22nd Annual IEEE Symposium on Logic in Computer Science, pages 213-224, Washington, DC, USA, 2007. IEEE Computer Society.

29. P.G. Jeavons. On the algebraic structure of combinatorial problems. Theoretical Computer Science, 200:185-204, 1998.

30. P.G. Jeavons, D.A. Cohen, and M.C. Cooper. Constraints, consistency and closure. Artificial Intelligence, 101(1-2):251-265, 1998.

31. P.G. Jeavons, D.A. Cohen, and M. Gyssens. Closure properties of constraints Journal of the ACM, 44:527-548, 1997.

32. P.G. Jeavons, D.A. Cohen, and J.K. Pearson. Constraints and universal algebra. Annals of Mathematics and Artificial Intelligence, 24:51-67, 1998.

33. B. Larose, C. Loten, and C. Tardif. A characterisation of first-order constraint satisfaction problems. In $L I C S$, pages 201-210, 2006.

34. B. Larose and P. Tesson. Universal algebra and hardness results for constraint satisfaction problems. In $I C A L P, 2007$.

35. B. Larose and L. Zadori. Bounded width problems and algebras. Algebra Universalis, 56(3-4):439-466, 2007.

36. Petar Marković and Ralph McKenzie. Few subpowers, congruence distributivity and near-unanimity. to appear in Algebra Universalis, 2006.

37. R.N. McKenzie, G.F. McNulty, and W.F. Taylor. Algebras, Lattices and Varieties, volume I. Wadsworth and Brooks, California, 1987.

38. Ralph McKenzie and Miklós Maróti. Existence theorems for weakly symmetric operations. to appear in Algebra Universalis, 2006.

39. T.J. Schaefer. The complexity of satisfiability problems. In Proceedings of the 10th ACM Symposium on Theory of Computing (STOC'78), pages 216-226, 1978.

40. A. Szendrei. Clones in Universal Algebra, volume 99 of Seminaires de Mathematiques Superieures. Université de Móntreal, 1986.

41. Matthew Valeriote. A subalgebra intersection property for congruence distributive varieties. Canadian Journal of Mathematics, accepted for publication, 2006. 